Bull. Fac .Agric., Cairo Univ.Vol. 68: 89-103 (2017).

\title{
A STUDY ON THE PLANT DIVERSITY IN ABU RAWASH AREA, GIZA, EGYPT
}

(Received:30.1.2017)

\author{
By \\ S. A. Azer \\ Flora and Phytotaxonomy Researches Department, Horticultural Research \\ Institute, Agricultural Research Center, Giza, Egypt
}

\begin{abstract}
The aim of the current research was to study plant diversity among seven represented habitats namely; field crops, orchards, irrigation canals, Mansuria Canal, deserts, drain station and water bodies in Abu Rawash area, Giza, Egypt. A total of 107 species belonging to 92 genera and 33 families were recorded and identified with regional flora and available checklists. The common families were Poaceae (22\%) followed by Compositae (12\%) Brassicaceae (7\%). Three species were recorded from each of Cyperaceae, Malvaceae and Zygophyllaceae, and two species from each of Amaranthaceae, Apiaceae, Apocynaceae, Plantaginaceae and Salicaceae. In addition, 16 families were monotypic. The annual species represented 53\%, perennial herbs were 35\% and each of shrubs and trees were $5 \%$; while biennials species were $2 \%$. The chorological ratios showed that the cosmopolitan taxa had the highest contribution (23\%), followed by pantropicals $(16 \%)$ and palaeotropicals $(13 \%)$. The cluster analysis divided the studied habitats into four groups. Group one included field crops and irrigation canals habitats. The second group contained orchards and Mansuria Canal habitats. The third group included drain station and deserts habitats; while the forth group contained water bodies' habitat. On the whole, orchards and Mansuria Canal habitats showed the highest similarity value (0.543). On the other hand, there was no similarity between species of water bodies' habitat and species of the other habitats except with Mansuria Canal (0.017).
\end{abstract}

Key words: Plant diversity, life cycle, life form, chorology, Abu Rawash.

\section{INTRODUCTION}

Egypt is characterized by a worm and almost rainless climate. The average annual rainfall over the whole country is only about 10 mm. Egypt's deserts occupy about $95 \%$ of its total area (Zahran and El-Amier, 2014). The Western desert covers approximately 700,000 $\mathrm{km}^{2}$, which is more than two-thirds of the total area of Egypt (Abd El-Ghani and Fawzy, 2006 and Salman et al., 2010). The Western desert consists of sandy plateau with some basins and depressions. It contains salty marshes, lakes, and waste lands (Boulos, 2008). The Precipitation decreases from $150 \mathrm{~mm}$ at the coast to zero in the south. It supports plants in areas with water resources such as rainfall and underground (Abd El-Ghani and Fawzy, 2006). Abu Rawash area belongs to Giza Governorate and located at the Western desert between latitude $30^{\circ} 02^{\prime} 48^{\prime \prime}$ and longitude $31^{\circ} 05^{\prime} 47^{\prime \prime}$. This area is strewn with sand and gravel of Oligocene. It represents one of the structural habitats that characterize the northern parts of Egypt (Kerdany and Cherif, 1990). In the Abu Rawash area, the rocks are mainly composed of limestones and dolomites. Topographically, the elevated upper cretaceous rocks are surrounded by younger tertiary rocks (Mansour, 2004).

The plant diversity in the Western desert of Egypt has been investigated by numerous studies such as; Täckholm \& Täckholm (1941); Täckholm and Drar (1950, 1954, 1969); Täckholm (1974),Boulos (1980, 1982a, \& b); Bornkamm (1986), Alaily et al. (1987), Kehl (1987); Bornkamm and Kehl (1989 \& 1990); Kehl and Bornkamm (1993); Boulos (1995, 1999, 2000, 2002, 2005 \& 2008); Boulos and Barakat (1998) and Azer (2013). Little information is known about the plant diversity in Abu Rawash area because of its unpredictable rain and the researchers reach the area after a prolonged drought and see old remnants of the vegetation. The aim of this study was to provide a description of the plant diversity and 
demonstrate life form and chorological ratios of the collected species among seven studied habitats namely; field crops, orchards, irrigation canals, Mansuria Canal, deserts, drain station and water bodies.

\section{MATERIALS AND METHODS \\ 2. 1. The Study Area}

Abu Rawash area belongs to Giza Governorate and located between latitude $30^{\circ}$ $02^{\prime} 48^{\prime \prime} \mathrm{N}$ and longitude $31^{\circ} 05^{\prime} 47^{\prime \prime}$ E. (Fig. 1). It is located in the Western desert and represents one of the structural habitats that characterize the northern parts of Egypt (Kerdany and Cherif, 1990). The area is composed of chalky limestone and dolomites (Faris \& Soliman, 1961). It is characterized by sand and gravel of Oligocene and Miocene age. The area is slightly subjected to tectonic activity during sedimentation of the rocks. Topographically, the elevated upper cretaceous rocks are surrounded by younger rocks (Mansour, 2004). The studied area was represented by seven different habitats namely; field crops, orchards, irrigation canals, Mansuria Canal, deserts, drain station and water bodies. Based on the climatic data, the mean annual temperature and rainfall are $26.25{ }^{\circ} \mathrm{C}$ and 1.25 $\mathrm{mm}$; respectively. Most of the rains fall in winter months (Table 1).

\section{2. Data Collection}

Field trips were performed to the study area to cover all the represented habitats from January 2016 to December 2016. Field data of the plant diversity, among seven represented habitats were gathered. The studied species were recorded to represent the plant diversity and covered the represented habitats in Abu Rawash area. The recorded species were arranged alphabetically with their families.

\subsection{Species identification}

Species identification was based on Täckholm (1974); Boulos (1995, 1999, 2000, 2002, 2005 \& 2009) and updated by Angiosperm Phylogeny Group III (2009) ; Chase and Reveal (2009) and Haston et al. (2009). Life forms (Therophytes, Geophytes, Chamaephytes, Hemicryptophytes, Nanophanerophytes, Phanerophytes, Hydrophytes) were identified according to Raunkiaer's system of classification (Raunkiaer 1934). Chorology (Cosmopolitan, Pantropical, Palaeotropical, Mediterranean, Irano-Turanian, Sudano-Zambezian, Euro-Siberian, SaharoSindian and cultivated) were cited according to Zohary (1966 \& 1987), Wickens (1976) and
Feinbrun-Dothan (1978 \& 1986). Voucher specimens were collected and identified at the herbarium of Flora and Phytotaxonomy Research Department (CAIM), Horticultural Research Institute, Agricultural Research Center, Giza, Egypt and arranged alphabetically with their families. 350 plant specimens were collected and prepared as herbarium sheets.

\section{4. Numerical analysis}

Numerical analysis was carried out and based on hierarchical cluster analysis. The retrieved output was used to construct specific ecological relationships among the studied habitats. The substantial numbers $(1=$ presence and $0=$ absence) of the recorded species were used for each selected habitat (Table 2). The data treated as a Pearson correlation in a data matrix to measure degree of similarity using SPSS version 22 (SPSS, 2013). The output was plotted in the form of dendrogram (Fig.4). The dendrogram was based on average linkage (between groups) and rescaled distance cluster combine

\section{1. Floristic composition}

\section{RESULTS}

The study recorded the presence of 107 species, representing 92 genera and 33 families belonged to dicotyledons families $(84 \%)$ and monocotyledons families (16\%) were identified (Fig.2). The most distributed families were Poaceae $(22 \%)$ followed by Compositae (12\%) and Brassicaceae (7\%). Moreover, each of Chenopodiaceae, Leguminosae, Polygonaceae, and Solanaceae was 5\%. Three species were recorded from each of Cyperaceae, Malvaceae and Zygophyllaceae and two species from each of Amaranthaceae, Apiaceae, Apocynaceae, Plantaginaceae and Salicaceae. Moreover, 16 monotypic families were represented by one species (Fig.3). The recorded species were arranged alphabetically with their families and genera and the presence or absence values in the studied habitats were listed in (Table 2).

\section{2 . Life cycle ratios}

The life cycle ratios of the studied species outlined in (Fig.5) revealed that the annual species dominated the flora of Abu Rawash area. This represented by 53\% followed by perennial herbs (35\%). Moreover, each of trees and shrubs was represented by $5 \%$; while biennials species were $2 \%$.

\section{3. Life form ratios}

The life forms of the collected taxa revealed that the therophytes species dominated the flora 
Table (1): Mean and annual values of temperature $\left({ }^{\circ} \mathrm{C}\right)$ and precipitation $(\mathrm{mm})$ of the study area during years 2015 \& 2016 (Based on Central Laboratory for Agricultural Climate data).

\begin{tabular}{|c|l|l|l|l|l|l|l|l|l|l|l|l|l|}
\hline Months & Jan. & Feb. & Mar. & Apr. & May. & Jun. & Jul. & Aug. & Sep. & Oct. & Nov. & Dec. & Annually \\
\hline Temperature & $\mathbf{1 7}$ & $\mathbf{1 9}$ & $\mathbf{2 2}$ & $\mathbf{2 7}$ & $\mathbf{3 1}$ & $\mathbf{3 3}$ & $\mathbf{3 3}$ & $\mathbf{3 3}$ & $\mathbf{3 1}$ & $\mathbf{2 8}$ & $\mathbf{2 3}$ & $\mathbf{1 8}$ & $(\mathbf{2 6 . 2 5})$ \\
\hline Precipitation & $\mathbf{4}$ & $\mathbf{3}$ & $\mathbf{2}$ & $\mathbf{0}$ & $\mathbf{0}$ & $\mathbf{0}$ & $\mathbf{0}$ & $\mathbf{0}$ & $\mathbf{0}$ & $\mathbf{0}$ & $\mathbf{2}$ & $\mathbf{4}$ & $(\mathbf{1 . 2 5})$ \\
\hline
\end{tabular}

Table (2): The life cycle, life form, chorology and data matrix of 107 species distributed among seven studied habitats in Abu Rawash area.

\begin{tabular}{|c|c|c|c|c|c|c|c|c|c|c|c|}
\hline \multirow[t]{2}{*}{ No } & & & & & \multicolumn{7}{|c|}{ Habitat types } \\
\hline & Taxa & 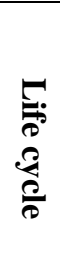 & 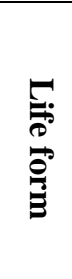 & 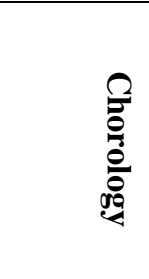 & 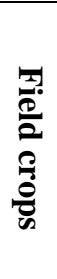 & 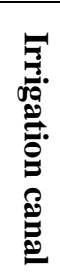 & 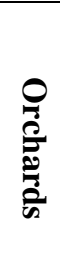 & 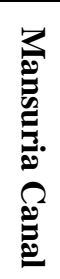 & 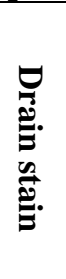 & 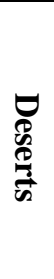 & 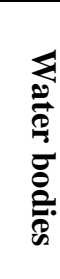 \\
\hline 1 & Aizoaceae & & & & & & & & & & \\
\hline 1.1 & Trianthema portulacastrum L. & A & $\mathrm{Th}$ & IT,SS & 1 & 1 & 1 & $\mathbf{0}$ & $\mathbf{0}$ & $\mathbf{0}$ & $\mathbf{0}$ \\
\hline 2 & Amaranthaceae & & & & & & & & & & \\
\hline 2.2 & Amaranthus blitum subsp. oleraceus (L.) Costea & A & $\mathrm{Th}$ & Cosm & 1 & 1 & 1 & 1 & $\mathbf{0}$ & $\mathbf{0}$ & $\mathbf{0}$ \\
\hline 2.3 & Amaranthus viridis $\mathrm{L}$. & A & Th & Pal & 1 & 1 & 1 & 1 & $\mathbf{0}$ & $\mathbf{0}$ & $\mathbf{0}$ \\
\hline 3 & Apiaceae & & & & & & & & & & \\
\hline 3.4 & Ammi majus L. & A & Th & SZ & 1 & 1 & $\mathbf{0}$ & $\mathbf{0}$ & $\mathbf{0}$ & $\mathbf{0}$ & $\mathbf{0}$ \\
\hline 4.5 & Cyclospermum leptophyllum (Pers.) Sprague & A & Th & Pal & 1 & 1 & $\mathbf{0}$ & $\mathbf{0}$ & $\mathbf{0}$ & $\mathbf{0}$ & $\mathbf{0}$ \\
\hline 4 & Apocynaceae & & & & & & & & & & \\
\hline 5.6 & Calotropis procera (Aiton) Dryand. & $\mathrm{P}$ & $\mathrm{Ph}$ & IT,SS,SZ & $\mathbf{0}$ & $\mathbf{0}$ & $\mathbf{0}$ & $\mathbf{0}$ & $\mathbf{0}$ & 1 & $\mathbf{0}$ \\
\hline 6.7 & Cynanchum acutum $\mathrm{L}$. & $\mathrm{P}$ & $\mathrm{Hm}$ & M,IT,SS & $\mathbf{0}$ & 1 & 1 & $\mathbf{0}$ & 1 & 1 & $\mathbf{0}$ \\
\hline 5 & Araceae & & & & & & & & & & \\
\hline 7.8 & Lemna gibba L. & $\mathrm{P}$ & Hy & M,IT,SS & $\mathbf{0}$ & $\mathbf{0}$ & $\mathbf{0}$ & $\mathbf{0}$ & $\mathbf{0}$ & $\mathbf{0}$ & 1 \\
\hline 6 & Arecaceae & & & & & & & & & & \\
\hline 8.9 & Phoenix dactylifera L. & $\mathrm{T}$ & $\mathrm{Ph}$ & M,IT & 1 & 1 & 1 & 1 & $\mathbf{0}$ & 1 & $\mathbf{0}$ \\
\hline 7 & Boraginaceae & & & & & & & & & & \\
\hline 9.10 & Heliotropium bacciferum Forssk. & $\mathrm{P}$ & $\mathrm{Ch}$ & $\mathrm{SS}$ & $\mathbf{0}$ & $\mathbf{0}$ & $\mathbf{0}$ & $\mathbf{0}$ & $\mathbf{0}$ & 1 & $\mathbf{0}$ \\
\hline 8 & Brassicaceae & & & & & & & & & & \\
\hline 10.11 & Brassica tournefortii Gouan & A & Th & M,IT & $\mathbf{0}$ & $\mathbf{0}$ & $\mathbf{0}$ & $\mathbf{0}$ & 1 & 1 & $\mathbf{0}$ \\
\hline 11.12 & Capsella bursa-pastoris (L.) Medik. & $\mathrm{A}$ & Th & Cosm & 1 & 1 & $\mathbf{0}$ & $\mathbf{0}$ & $\mathbf{0}$ & $\mathbf{0}$ & $\mathbf{0}$ \\
\hline 12.13 & Eruca vesicaria (L.) Cav. & $\mathrm{A}$ & Th & M & 1 & $\mathbf{0}$ & 1 & 1 & $\mathbf{0}$ & $\mathbf{0}$ & $\mathbf{0}$ \\
\hline 13.14 & Lepidium sativum L. & $\mathrm{A}$ & Th & Cosm & 1 & 1 & $\mathbf{0}$ & $\mathbf{0}$ & $\mathbf{0}$ & $\mathbf{0}$ & $\mathbf{0}$ \\
\hline 14.15 & Raphanus raphanistrum L. & A & Th & Cosm & 1 & $\mathbf{0}$ & 1 & $\mathbf{0}$ & $\mathbf{0}$ & $\mathbf{0}$ & $\mathbf{0}$ \\
\hline 15.16 & Sinapis alba $\mathrm{L}$. & $\mathrm{A}$ & Th & Pan & $\mathbf{0}$ & $\mathbf{0}$ & $\mathbf{0}$ & $\mathbf{0}$ & 1 & 1 & $\mathbf{0}$ \\
\hline 16.17 & Sisymbrium irio L. & $\mathrm{A}$ & $\mathrm{Th}$ & M,IT,ES & 1 & $\mathbf{0}$ & $\mathbf{0}$ & 1 & 1 & 1 & $\mathbf{0}$ \\
\hline 9 & Ceratophyllaceae & & & & & & & & & & \\
\hline 17.18 & Ceratophyllum demersum L. & $\mathrm{P}$ & Hy & Cosm & $\mathbf{0}$ & $\mathbf{0}$ & $\mathbf{0}$ & $\mathbf{0}$ & $\mathbf{0}$ & $\mathbf{0}$ & 1 \\
\hline 10 & Chenopodiaceae & & & & & & & & & & \\
\hline 18.19 & Bassia indica (Wight) A.J.Scott & $\mathrm{A}$ & $\mathrm{Th}$ & It,SS & $\mathbf{0}$ & $\mathbf{0}$ & $\mathbf{0}$ & 1 & 1 & 1 & $\mathbf{0}$ \\
\hline 19.20 & Beta vulgaris $\mathrm{L}$. & $\mathrm{A}$ & Th & SS & 1 & 1 & $\mathbf{0}$ & $\mathbf{0}$ & $\mathbf{0}$ & $\mathbf{0}$ & $\mathbf{0}$ \\
\hline 20.21 & Chenopodium album L. & $\mathrm{A}$ & $\mathrm{Th}$ & Cosm & 1 & 1 & 1 & 1 & 1 & $\mathbf{0}$ & $\mathbf{0}$ \\
\hline 20.22 & Chenopodium murale L. & $\mathrm{A}$ & Th & Cosm & 1 & 1 & 1 & 1 & 1 & $\mathbf{0}$ & $\mathbf{0}$ \\
\hline 21.23 & Suaeda vermiculata Forssk. ex J.F.Gmel. & $\mathrm{S}$ & $\mathrm{Ch}$ & SZ & $\mathbf{0}$ & $\mathbf{0}$ & $\mathbf{0}$ & 1 & 1 & 1 & $\mathbf{0}$ \\
\hline 11 & Compositae & & & & & & & & & & \\
\hline 22.24 & Achillea tenuifolia Lam. & $\mathrm{P}$ & $\mathrm{Na}$ & SS & $\mathbf{0}$ & $\mathbf{0}$ & $\mathbf{0}$ & $\mathbf{0}$ & $\mathbf{0}$ & 1 & $\mathbf{0}$ \\
\hline 23.25 & Artemisia judaica $\mathrm{L}$. & $\mathrm{P}$ & $\mathrm{Ch}$ & $\mathrm{M}, \mathrm{SS}$ & $\mathbf{0}$ & $\mathbf{0}$ & $\mathbf{0}$ & $\mathbf{0}$ & $\mathbf{0}$ & 1 & $\mathbf{0}$ \\
\hline 24.26 & Bidens pilose L. & $\mathrm{A}$ & Th & Pan & $\mathbf{0}$ & 1 & 1 & 1 & $\mathbf{0}$ & $\mathbf{0}$ & $\mathbf{0}$ \\
\hline 25.27 & $\begin{array}{l}\text { Cichorium endivia L. subsp. divaricatum } \\
\text { (Schousb.) P.D. Sell }\end{array}$ & A & Th & M,IT & 1 & 1 & 1 & $\mathbf{0}$ & $\mathbf{0}$ & $\mathbf{0}$ & $\mathbf{0}$ \\
\hline 26.28 & Eclipta prostrata $(\mathrm{L}.) \mathrm{L}$. & A & Th & Pan & $\mathbf{0}$ & 1 & $\mathbf{0}$ & 1 & $\mathbf{0}$ & $\mathbf{0}$ & $\mathbf{0}$ \\
\hline 27.29 & Erigeron bonariensis L. & A & Th & $\mathrm{M}$ & $\mathbf{0}$ & 1 & 1 & 1 & 1 & $\mathbf{0}$ & $\mathbf{0}$ \\
\hline
\end{tabular}




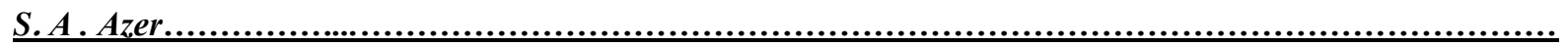

Table (2): Continued I

\begin{tabular}{|c|c|c|c|c|c|c|c|c|c|c|c|}
\hline No & & & & & & & & itat 1 & ypes & & \\
\hline & Taxa & 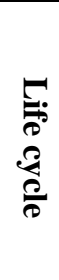 & $\begin{array}{l}\stackrel{\overrightarrow{0}}{\circ} \\
\stackrel{0}{0} \\
\stackrel{0}{\Xi}\end{array}$ & 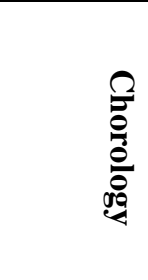 & 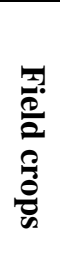 & 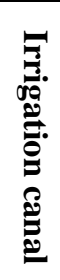 & 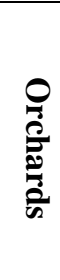 & 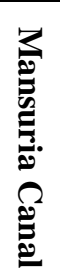 & 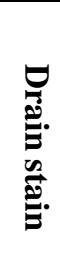 & 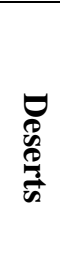 & 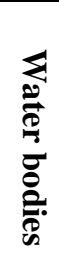 \\
\hline 28.30 & Lactuca serriola L. & $\mathrm{B}$ & Th & Cosm & $\mathbf{0}$ & $\mathbf{0}$ & 1 & 1 & 1 & 1 & $\mathbf{0}$ \\
\hline 29.31 & Launaea nudicaulis (L.) Hook.f. & $\mathrm{P}$ & $\mathrm{Ch}$ & Cosm & $\mathbf{0}$ & $\mathbf{0}$ & $\mathbf{0}$ & 1 & 1 & 1 & $\mathbf{0}$ \\
\hline 30.32 & Pluchea dioscoridis (L.) DC. & $\mathrm{S}$ & $\mathrm{Ch}$ & Cosm & $\mathbf{0}$ & $\mathbf{0}$ & $\mathbf{0}$ & $\mathbf{0}$ & 1 & 1 & $\mathbf{0}$ \\
\hline 31.33 & Senecio aegyptius $\mathrm{L}$. & A & Th & $\mathrm{M}$ & $\mathbf{0}$ & $\mathbf{0}$ & $\mathbf{0}$ & $\mathbf{0}$ & 1 & 1 & $\mathbf{0}$ \\
\hline 31.34 & Senecio desfontainei Druce & A & Th & SZ & $\mathbf{0}$ & $\mathbf{0}$ & $\mathbf{0}$ & $\mathbf{0}$ & 1 & 1 & $\mathbf{0}$ \\
\hline 32.35 & $\begin{array}{l}\text { Sericocarpus linifolius (L.) "Britton, Sterns } \\
\text { \& Poggenb." }\end{array}$ & $\bar{A}$ & Th & M,IT & $\mathbf{0}$ & $\mathbf{0}$ & 1 & $\mathbf{0}$ & $\mathbf{0}$ & 1 & $\mathbf{0}$ \\
\hline 33.36 & Sonchus oleraceus (L.) L. & A & Th & Cosm & 1 & 1 & 1 & 1 & $\mathbf{0}$ & $\mathbf{0}$ & $\mathbf{0}$ \\
\hline 12 & Convolvulaceae & & & & & & & & & & \\
\hline 34.37 & Convolvulus arvensis $\mathrm{L}$. & $\mathrm{P}$ & $\mathrm{Ch}$ & Pal & 1 & 1 & 1 & 1 & 1 & $\mathbf{0}$ & $\mathbf{0}$ \\
\hline 35.38 & Cuscuta campestris Yunck. & A & Th & Pan & 1 & 1 & $\mathbf{0}$ & $\mathbf{0}$ & $\mathbf{0}$ & $\mathbf{0}$ & $\mathbf{0}$ \\
\hline 36.39 & Ipomoea carnea Jacq. & $\mathrm{S}$ & $\mathrm{Ph}$ & Pan & $\mathbf{0}$ & $\mathbf{0}$ & 1 & 1 & $\mathbf{0}$ & $\mathbf{0}$ & $\mathbf{0}$ \\
\hline 36.40 & Ipomoea cairica $(\mathrm{L}$.) Sweet & $\mathrm{P}$ & $\mathrm{Ch}$ & Pan & $\mathbf{0}$ & $\mathbf{0}$ & $\mathbf{1}$ & 1 & $\mathbf{0}$ & $\mathbf{0}$ & $\mathbf{0}$ \\
\hline 13 & Cyperaceae & & & & & & & & & & \\
\hline 37.41 & Cyperus alopecuroides Rottb. & $\mathrm{P}$ & $\mathrm{Ch}$ & Pan & $\mathbf{0}$ & $\mathbf{0}$ & $\mathbf{0}$ & 1 & $\mathbf{0}$ & $\mathbf{0}$ & $\mathbf{0}$ \\
\hline 37.42 & Cyperus articulatus $\mathrm{L}$. & $\mathrm{P}$ & $\mathrm{Ch}$ & Pal & $\mathbf{0}$ & $\mathbf{0}$ & $\mathbf{0}$ & 1 & $\mathbf{0}$ & $\mathbf{0}$ & $\mathbf{0}$ \\
\hline 37.43 & Cyperus rotundus L. & $\mathrm{P}$ & $\mathrm{Ge}$ & Pan & 1 & 1 & 1 & 1 & 1 & $\mathbf{0}$ & $\mathbf{0}$ \\
\hline 14 & Euphorbiaceae & & & & & & & & & & \\
\hline 38.44 & Euphorbia heterophylla L. & A & Th & Cosm & 1 & 1 & $\mathbf{1}$ & 1 & $\mathbf{0}$ & $\mathbf{0}$ & $\mathbf{0}$ \\
\hline 38.45 & Euphorbia peplus L. & A & Th & Pan & 1 & 1 & 1 & 1 & $\mathbf{0}$ & $\mathbf{0}$ & $\mathbf{0}$ \\
\hline 38.46 & Euphorbia prostrata Aiton & A & Th & Cosm & $\mathbf{0}$ & 1 & $\mathbf{0}$ & $\mathbf{0}$ & $\mathbf{0}$ & $\mathbf{0}$ & $\mathbf{0}$ \\
\hline 39.47 & Ricinus communis L. & $\mathrm{S}$ & $\mathrm{Ph}$ & $\mathrm{M}$ & $\mathbf{0}$ & $\mathbf{0}$ & 1 & 1 & 1 & 1 & $\mathbf{0}$ \\
\hline 15 & Lamiaceae & & & & & & & & & & \\
\hline 40.48 & $\begin{array}{l}\text { Mentha longifolia subsp. typhoides (Briq.) } \\
\text { Harley }\end{array}$ & $\mathrm{P}$ & $\mathrm{Ch}$ & Pal & $\mathbf{0}$ & 1 & $\mathbf{0}$ & $\mathbf{0}$ & $\mathbf{0}$ & $\mathbf{0}$ & $\mathbf{0}$ \\
\hline 16 & Leguminosae & & & & & & & & & & \\
\hline 41.49 & Alhagi graecorum Boiss. & $\mathrm{P}$ & $\mathrm{Hm}$ & $\mathrm{Pal}$ & $\mathbf{0}$ & $\mathbf{0}$ & 1 & 1 & 1 & 1 & $\mathbf{0}$ \\
\hline 42.50 & Leucaena leucocephala (Lam.) De Wit & $\mathrm{T}$ & $\mathrm{Ph}$ & Pal & $\mathbf{0}$ & $\mathbf{0}$ & $\mathbf{0}$ & 1 & $\mathbf{0}$ & $\mathbf{0}$ & $\mathbf{0}$ \\
\hline 43.51 & Melilotus indicus (L.) All. & A & Th & M,ES,SS & 1 & $\mathbf{0}$ & $\mathbf{0}$ & $\mathbf{0}$ & 1 & $\mathbf{0}$ & $\mathbf{0}$ \\
\hline 44.52 & Sesbania sesban (L.) Merr. & $\mathrm{S}$ & $\mathrm{Ph}$ & M,IT & 1 & $\mathbf{1}$ & 1 & 1 & 1 & 1 & $\mathbf{0}$ \\
\hline 45.53 & Trifolium resupinatum $\mathrm{L}$. & A & Th & Pal & 1 & $\mathbf{0}$ & 1 & 1 & 1 & $\mathbf{0}$ & $\mathbf{0}$ \\
\hline 17 & Malvaceae & & & & & & & & & & \\
\hline 46.54 & Corchorus olitorius L. & A & Th & Pan & 1 & 1 & 1 & 1 & $\mathbf{0}$ & $\mathbf{0}$ & $\mathbf{0}$ \\
\hline 47.55 & Malva parviflora $\mathrm{L}$. & A & Th & M,IT & 1 & 1 & 1 & 1 & 1 & $\mathbf{0}$ & $\mathbf{0}$ \\
\hline 48.56 & Sida spinosa $\mathrm{L}$. & $\mathrm{P}$ & $\mathrm{Ch}$ & Cosm & $\mathbf{0}$ & $\mathbf{0}$ & 1 & $\mathbf{0}$ & $\mathbf{0}$ & $\mathbf{0}$ & $\mathbf{0}$ \\
\hline 18 & Nyctaginaceae & & & & & & & & & & \\
\hline 49.57 & Boerhavia coccinea Mill. & $\mathrm{P}$ & $\mathrm{Hm}$ & Cosm & $\mathbf{0}$ & $\mathbf{0}$ & $\mathbf{0}$ & $\mathbf{0}$ & 1 & $\mathbf{0}$ & $\mathbf{0}$ \\
\hline 19 & Onagraceae & & & & & & & & & & \\
\hline 50.58 & $\begin{array}{l}\text { Ludwigia adscendens subsp. diffusa (Forssk.) } \\
\text { P.H.Raven }\end{array}$ & $\mathrm{P}$ & $\mathrm{He}$ & M,ES,SS & $\mathbf{0}$ & $\mathbf{0}$ & $\mathbf{0}$ & $\mathbf{0}$ & $\mathbf{0}$ & $\mathbf{0}$ & 1 \\
\hline 20 & Oxalidaceae & & & & & & & & & & \\
\hline 51.59 & Oxalis corniculata $\mathrm{L}$. & $\mathrm{P}$ & $\mathrm{Hm}$ & Pal & $\mathbf{0}$ & $\mathbf{0}$ & 1 & $\mathbf{0}$ & $\mathbf{0}$ & $\mathbf{0}$ & $\mathbf{0}$ \\
\hline 21 & Plantaginaceae & & & & & & & & & & \\
\hline 52.60 & Plantago lagopus $\mathrm{L}$. & A & Th & Pan & $\mathbf{0}$ & $\mathbf{0}$ & $\mathbf{1}$ & $\mathbf{0}$ & $\mathbf{0}$ & $\mathbf{0}$ & $\mathbf{0}$ \\
\hline 52.61 & Plantago major $\mathrm{L}$. & A & Th & M,IT & 1 & 1 & 1 & $\mathbf{0}$ & $\mathbf{0}$ & $\mathbf{0}$ & $\mathbf{0}$ \\
\hline
\end{tabular}


Table (2): Continued II

\begin{tabular}{|c|c|c|c|c|c|c|c|c|c|c|c|}
\hline No & & & & & & & Ha & itat & pes & & \\
\hline & Taxa & $\begin{array}{l}\stackrel{1}{0} \\
\stackrel{0}{0} \\
\stackrel{3}{0} \\
\frac{0}{2}\end{array}$ & 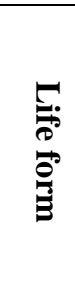 & 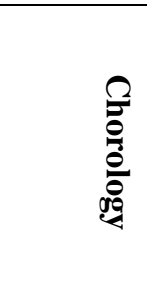 & 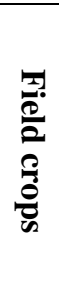 & 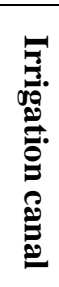 & 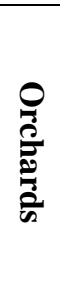 & 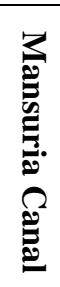 & 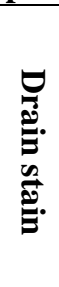 & 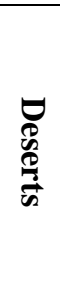 & 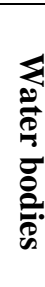 \\
\hline 22 & Poaceae & & & & & & & & & & \\
\hline 53.62 & Arundo donax L. & $\mathrm{P}$ & $\mathrm{He}$ & $\mathrm{C}$ & $\mathbf{0}$ & $\mathbf{0}$ & $\mathbf{0}$ & $\mathbf{0}$ & $\mathbf{0}$ & 1 & $\mathbf{0}$ \\
\hline 54.63 & Avena sativa $\mathrm{L}$. & $\mathrm{A}$ & Th & $\mathrm{C}$ & $\mathbf{0}$ & $\mathbf{0}$ & $\mathbf{0}$ & $\mathbf{0}$ & $\mathbf{0}$ & $\mathbf{0}$ & $\mathbf{0}$ \\
\hline 54.64 & Avena barbata Pott ex Link & $\mathrm{A}$ & Th & M,IT & $\mathbf{0}$ & $\mathbf{0}$ & $\mathbf{0}$ & $\mathbf{0}$ & 1 & 1 & $\mathbf{0}$ \\
\hline 55.65 & Brachiaria mutica (Forssk.) Stapf & A & Th & Cosm & 1 & 1 & 1 & $\mathbf{0}$ & $\mathbf{0}$ & $\mathbf{0}$ & $\mathbf{0}$ \\
\hline 56.66 & Bromus catharticus Vahl & $\mathrm{A}$ & Th & Cosm & $\mathbf{0}$ & $\mathbf{0}$ & $\mathbf{0}$ & $\mathbf{1}$ & 1 & 1 & $\mathbf{0}$ \\
\hline 57.67 & Cenchrus biflorus Roxb. & $\mathrm{A}$ & Th & SS,SZ & $\mathbf{0}$ & $\mathbf{0}$ & 1 & 1 & $\mathbf{0}$ & $\mathbf{0}$ & $\mathbf{0}$ \\
\hline 58.68 & Cynodon dactylon (L.) Pers. & $\mathrm{P}$ & $\mathrm{Ge}$ & IT,SS,SZ & 1 & 1 & 1 & 1 & 1 & 1 & $\mathbf{0}$ \\
\hline 59.69 & Desmostachya bipinnata (L.) Stapf & $\mathrm{P}$ & $\mathrm{Ge}$ & SS,SZ & $\mathbf{0}$ & $\mathbf{0}$ & $\mathbf{0}$ & 1 & $\mathbf{0}$ & $\mathbf{0}$ & $\mathbf{0}$ \\
\hline 60.70 & Digitaria sanguinalis (L.) Scop. & A & Th & Pal & $\mathbf{0}$ & 1 & 1 & 1 & 1 & 1 & $\mathbf{0}$ \\
\hline 61.71 & Echinochloa colona (L.) Link & A & Th & Pan & 1 & 1 & 1 & 1 & $\mathbf{0}$ & $\mathbf{0}$ & $\mathbf{0}$ \\
\hline 61.72 & Echinochloa stagnina (Retz.) P. Beauv. & $\mathrm{P}$ & $\mathrm{Ge}$ & Pal & $\mathbf{0}$ & 1 & 1 & 1 & $\mathbf{0}$ & $\mathbf{0}$ & $\mathbf{0}$ \\
\hline 62.73 & Imperata cylindrica (L.) Raeusch. & $\mathrm{P}$ & $\mathrm{Hm}$ & SS,SZ & $\mathbf{0}$ & $\mathbf{0}$ & $\mathbf{0}$ & 1 & 1 & 1 & $\mathbf{0}$ \\
\hline 63.74 & Leptochloa fusca (L.) Kunth & $\mathrm{P}$ & $\mathrm{Ch}$ & $\mathrm{SZ}$ & $\mathbf{0}$ & 1 & 1 & $\mathbf{0}$ & $\mathbf{0}$ & $\mathbf{0}$ & $\mathbf{0}$ \\
\hline 64.75 & Lolium multiflorum Lam. & $\mathrm{A}$ & Th & Pan & $\mathbf{0}$ & $\mathbf{1}$ & 1 & 1 & 1 & 1 & $\mathbf{0}$ \\
\hline 65.76 & Paspalidium geminatum (Forssk.) Stapf & $\mathrm{P}$ & $\mathrm{Ch}$ & Cosm & $\mathbf{0}$ & 1 & 1 & 1 & $\mathbf{0}$ & $\mathbf{0}$ & $\mathbf{0}$ \\
\hline 66.77 & $\begin{array}{l}\text { Pennisetum divisum (Forrsk.) ex J.F. } \\
\text { Gmel.) Henrard }\end{array}$ & $\mathrm{P}$ & $\mathrm{Ch}$ & $\mathrm{Pal}$ & $\mathbf{0}$ & $\mathbf{0}$ & $\mathbf{0}$ & $\mathbf{0}$ & $\mathbf{0}$ & 1 & $\mathbf{0}$ \\
\hline 67.78 & Phalaris minor Retz. & A & Th & Pan & $\mathbf{0}$ & $\mathbf{0}$ & 1 & $\mathbf{0}$ & $\mathbf{0}$ & $\mathbf{0}$ & $\mathbf{0}$ \\
\hline 68.79 & $\begin{array}{l}\text { Phragmites australis (Cav.) Trin. ex } \\
\text { Steud. }\end{array}$ & $P$ & $\mathrm{Hm}$ & $\mathrm{SS}, \mathrm{SZ}$ & $\mathbf{0}$ & $\mathbf{0}$ & $\mathbf{0}$ & 1 & $\mathbf{0}$ & 1 & $\mathbf{0}$ \\
\hline 69.80 & Pоа аппиа $\mathrm{L}$. & A & Th & M,IT,SS & 1 & $\mathbf{0}$ & 1 & $\mathbf{0}$ & $\mathbf{0}$ & $\mathbf{0}$ & $\mathbf{0}$ \\
\hline 70.81 & Polypogon monspeliensis (L.) Desf. & A & Th & M,IT,ES & $\mathbf{0}$ & 1 & 1 & 1 & 1 & $\mathbf{0}$ & $\mathbf{0}$ \\
\hline 71.82 & Saccharum spontaneum L. & $\mathrm{P}$ & $\mathrm{Hm}$ & $\mathrm{M}, \mathrm{SS}$ & $\mathbf{0}$ & $\mathbf{0}$ & 1 & 1 & $\mathbf{0}$ & $\mathbf{0}$ & $\mathbf{0}$ \\
\hline 72.83 & Setaria verticillata (L.) P. Beauv. & A & Th & SZ & $\mathbf{0}$ & $\mathbf{0}$ & 1 & 1 & $\mathbf{0}$ & $\mathbf{0}$ & $\mathbf{0}$ \\
\hline 72.84 & Setaria viridis (L.) P. Beauv. & A & Th & Cosm & $\mathbf{0}$ & $\mathbf{0}$ & 1 & 1 & $\mathbf{0}$ & $\mathbf{0}$ & $\mathbf{0}$ \\
\hline 73.85 & Sorghum virgatum (Hack.) Stapf & $\mathrm{B}$ & Th & $\mathrm{C}$ & $\mathbf{0}$ & $\mathbf{0}$ & 1 & $\mathbf{0}$ & $\mathbf{0}$ & $\mathbf{0}$ & $\mathbf{0}$ \\
\hline 23 & Polygonaceae & & & & & & & & & & \\
\hline 74.86 & Calligonum comosum L'Hér. & $\mathrm{P}$ & $\mathrm{Ch}$ & IT,SS & $\mathbf{0}$ & $\mathbf{0}$ & $\mathbf{0}$ & $\mathbf{0}$ & $\mathbf{0}$ & 1 & $\mathbf{0}$ \\
\hline 75.87 & Emex spinosa (L.) Campd. & A & Th & M,IT,SS & $\mathbf{0}$ & 1 & 1 & $\mathbf{0}$ & $\mathbf{0}$ & $\mathbf{0}$ & $\mathbf{0}$ \\
\hline 76.88 & Persicaria senegalensis (Meisn.) Soják & $\mathrm{P}$ & $\mathrm{Hm}$ & SS & $\mathbf{0}$ & $\mathbf{0}$ & $\mathbf{0}$ & $\mathbf{0}$ & $\mathbf{0}$ & $\mathbf{0}$ & 1 \\
\hline 77.89 & Rumex dentatus $\mathrm{L}$. & A & Th & Cosm & $\mathbf{0}$ & $\mathbf{0}$ & 1 & $\mathbf{0}$ & 1 & 1 & $\mathbf{0}$ \\
\hline 77.90 & Rumex vesicarius $\mathrm{L}$. & A & Th & M,IS,ES & $\mathbf{0}$ & $\mathbf{0}$ & $\mathbf{0}$ & $\mathbf{0}$ & $\mathbf{0}$ & 1 & $\mathbf{0}$ \\
\hline 24 & Pontederiaceae & & & & & & & & & & \\
\hline 78.91 & Eichhornia crassipes (Mart.) Solms & $\mathrm{P}$ & $\mathrm{Hy}$ & Pal & $\mathbf{0}$ & $\mathbf{0}$ & $\mathbf{0}$ & 1 & $\mathbf{0}$ & $\mathbf{0}$ & 1 \\
\hline 25 & Portulacaceae & & & & & & & & & & \\
\hline 79.92 & Portulaca oleracea L. & A & Th & Cosm & 1 & $\mathbf{0}$ & 1 & 1 & 1 & $\mathbf{0}$ & $\mathbf{0}$ \\
\hline 26 & Potamogetonaceae & & & & & & & & & & \\
\hline 80.93 & Potamogeton nodosus Poir. & $\mathrm{P}$ & $\mathrm{Hy}$ & Cosm & $\mathbf{0}$ & $\mathbf{0}$ & $\mathbf{0}$ & $\mathbf{0}$ & $\mathbf{0}$ & $\mathbf{0}$ & 1 \\
\hline 27 & Primulaceae & & & & & & & & & & \\
\hline 81.94 & Anagallis arvensis L. & A & Th & Cosm & 1 & $\mathbf{0}$ & $\mathbf{0}$ & $\mathbf{0}$ & 1 & $\mathbf{0}$ & $\mathbf{0}$ \\
\hline 28 & Salicaceae & & & & & & & & & & \\
\hline 82.95 & Salix mucronata Thunb. & $\mathrm{T}$ & $\mathrm{Ph}$ & Pan & $\mathbf{0}$ & $\mathbf{0}$ & $\mathbf{0}$ & 1 & $\mathbf{0}$ & $\mathbf{0}$ & $\mathbf{0}$ \\
\hline 82.96 & Salix tetrasperma Roxb. & $\mathrm{T}$ & $\mathrm{Ph}$ & $\mathrm{Pal}$ & $\mathbf{0}$ & $\mathbf{0}$ & 1 & 1 & $\mathbf{0}$ & $\mathbf{0}$ & $\mathbf{0}$ \\
\hline
\end{tabular}


Table (2): Continued III

\begin{tabular}{|c|c|c|c|c|c|c|c|c|c|c|c|}
\hline No & & & & & \multicolumn{7}{|c|}{ Habitat types } \\
\hline & Taxa & $\begin{array}{l}5 \\
\frac{0}{0} \\
\frac{2}{2} \\
\frac{2}{2}\end{array}$ & $\begin{array}{l}\stackrel{5}{\overrightarrow{0}} \\
\stackrel{0}{0} \\
\stackrel{3}{B}\end{array}$ & 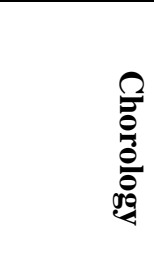 & $\begin{array}{l}3 \\
\frac{3}{2} \\
\frac{3}{2} \\
0 \\
0\end{array}$ & 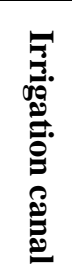 & 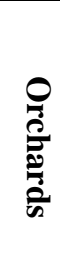 & 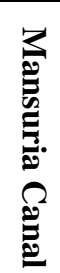 & 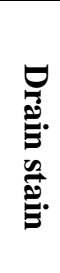 & 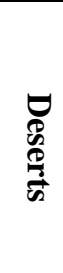 & 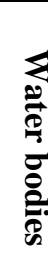 \\
\hline 29 & Solanaceae & & & & & & & & & & \\
\hline 83.97 & Datura stramonium L. & A & Th & Pan & $\mathbf{0}$ & $\mathbf{0}$ & $\mathbf{0}$ & 1 & 1 & $\mathbf{0}$ & $\mathbf{0}$ \\
\hline 84.98 & Lycopersicon esculentum Mill. & $\mathrm{A}$ & Th & $\mathrm{C}$ & 1 & $\mathbf{0}$ & 1 & 1 & $\mathbf{0}$ & $\mathbf{0}$ & $\mathbf{0}$ \\
\hline 85.99 & Solanum americanum Mill. & $\mathrm{A}$ & $\mathrm{Th}$ & Cosm & 1 & 1 & $\mathbf{1}$ & 1 & $\mathbf{0}$ & $\mathbf{0}$ & $\mathbf{0}$ \\
\hline 86.100 & Solanum tuberosum $\mathrm{L}$. & A & Th & Cosm & $\mathbf{1}$ & 1 & $\mathbf{0}$ & $\mathbf{0}$ & $\mathbf{0}$ & $\mathbf{0}$ & $\mathbf{0}$ \\
\hline 87.101 & Withania somnifera (L.) Dunal & $\mathrm{P}$ & $\mathrm{Ch}$ & SS,SZ & $\mathbf{0}$ & 1 & 1 & 1 & 1 & $\mathbf{1}$ & $\mathbf{0}$ \\
\hline 30 & Tamaricaceae & & & & & & & & & & \\
\hline 88.102 & Tamarix senegalensis DC. & $\mathrm{T}$ & $\mathrm{Ph}$ & M,ES,SS & $\mathbf{0}$ & $\mathbf{0}$ & $\mathbf{0}$ & $\mathbf{0}$ & 1 & 1 & $\mathbf{0}$ \\
\hline 31 & Typhaceae & & & & & & & & & & \\
\hline 89.103 & Typha domingensis Pers. & $\mathrm{P}$ & $\mathrm{Hm}$ & M,IS,ES & $\mathbf{0}$ & $\mathbf{0}$ & $\mathbf{0}$ & $\mathbf{0}$ & $\mathbf{0}$ & 1 & $\mathbf{0}$ \\
\hline 32 & Urticaceae & & & & & & & & & & \\
\hline 90.104 & Urtica urens L. & A & $\mathrm{Th}$ & Pan & 1 & $\mathbf{0}$ & 1 & $\mathbf{0}$ & $\mathbf{0}$ & $\mathbf{0}$ & $\mathbf{0}$ \\
\hline 33 & Zygophyllaceae & & & & & & & & & & \\
\hline 91.105 & Fagonia mollis Delile & $\mathrm{P}$ & $\mathrm{Ch}$ & Cosm & $\mathbf{0}$ & $\mathbf{0}$ & $\mathbf{0}$ & $\mathbf{0}$ & $\mathbf{0}$ & $\mathbf{1}$ & $\mathbf{0}$ \\
\hline 92.106 & Zygophyllum album L.f. & $\mathrm{P}$ & $\mathrm{Ch}$ & IT,SS,SZ & $\mathbf{0}$ & $\mathbf{0}$ & $\mathbf{0}$ & $\mathbf{0}$ & $\mathbf{0}$ & 1 & $\mathbf{0}$ \\
\hline 92.107 & Zygophyllum simplex L. & $\mathrm{A}$ & Th & M,SS & $\mathbf{0}$ & $\mathbf{0}$ & $\mathbf{0}$ & $\mathbf{0}$ & $\mathbf{0}$ & 1 & $\mathbf{0}$ \\
\hline
\end{tabular}

$\mathrm{A}=$ annual, $\mathrm{B}=$ biennial, $\mathrm{P}=$ perennial herb, $\mathrm{S}=$ shrub, $\mathrm{T}=$ tree, $\mathrm{Th}=$ Therophytes, $\mathrm{Hy}=\mathrm{Hydrophytes}, \mathrm{Hm}=$ Hemicryptophytes, $\mathrm{Na}=$ Nanophanerophytes, $\mathrm{Ph}=$ Phanerophytes, $\mathrm{Ch}=$ Chaemophytes, $\mathrm{Ge}=\mathrm{Geophytes}$, $\mathrm{He}=$ Helophyte, Cosm=Cosmopolitan, $\mathbf{P a n}=$ Pantropical, Pal= Palaeotropical, $\mathrm{M}=$ Mediterranean, $\mathbf{I T}=$ Irano-Turanian, $\mathbf{C}=$ Cultivated, $\mathbf{S Z}=$ Sudano-Zambezian, $\mathbf{S S}=$ Saharo-Sindian,

ES= Euro-Siberian, $1=$ presence, $0=$ absence.

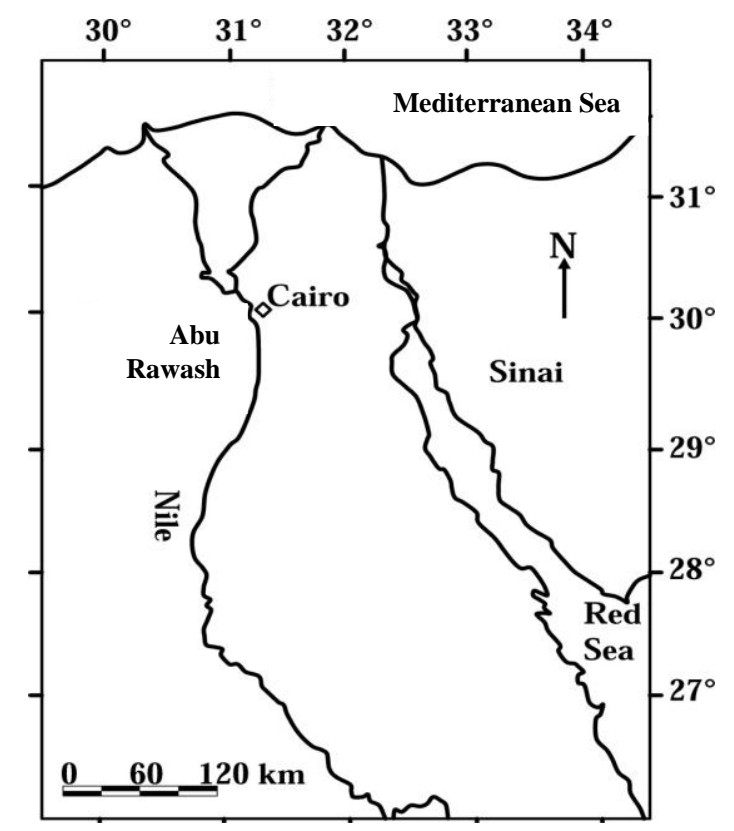

Fig.(1): A map showing the location of Abu Rawash area at Giza Governorate, Egypt. 


\section{Angiospermae species ratios}

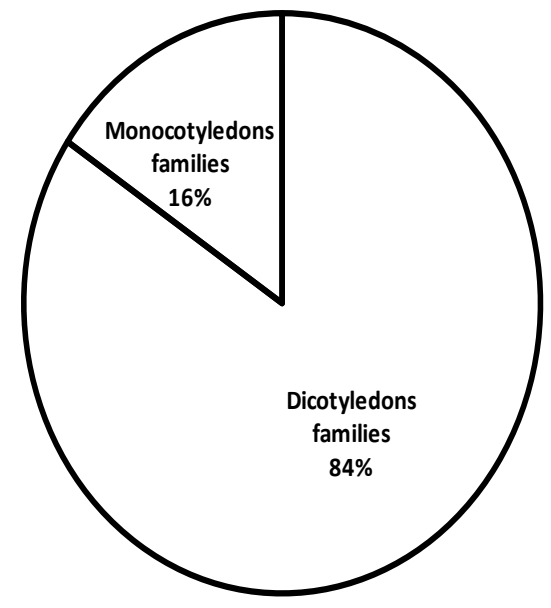

Fig. (2): Spectrum showing the total ratios of dicotyledons and monocotyledons families in Abu Rawash area.

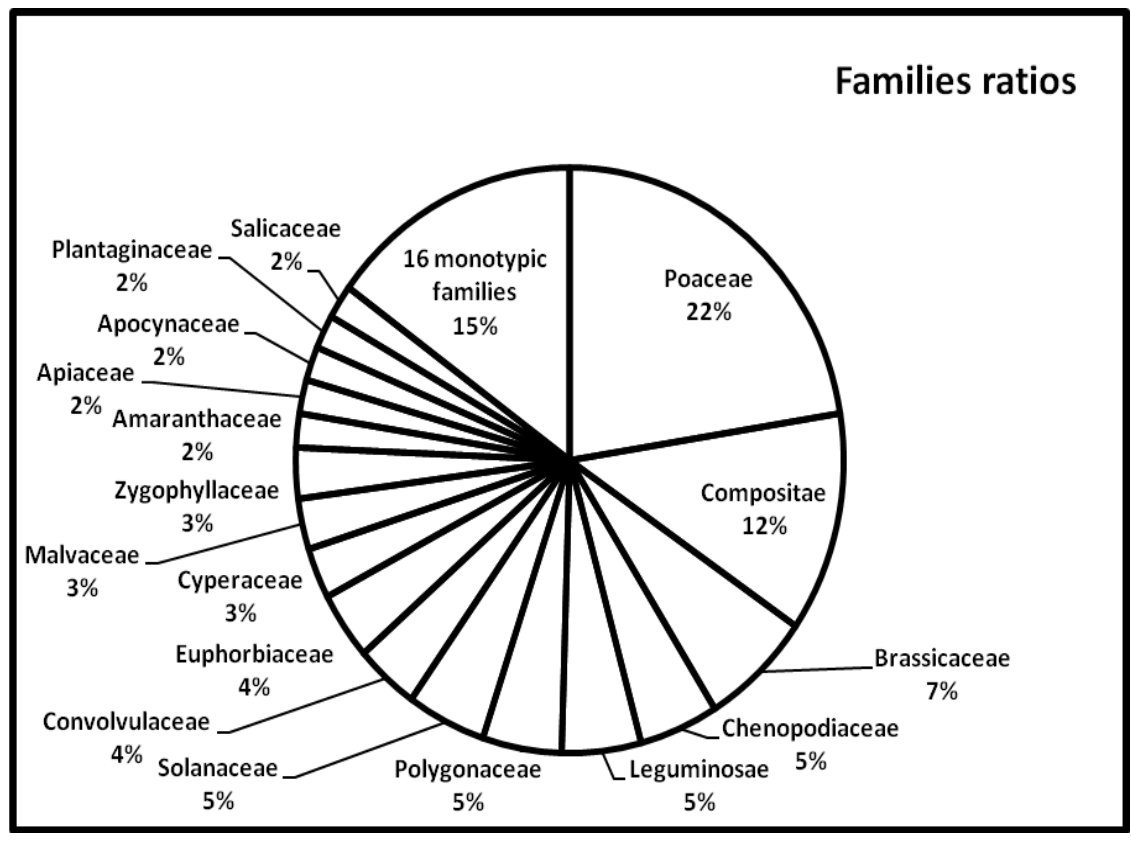

Fig. (3): Spectrum showing the ratios of the recorded families in Abu Rawash area. 


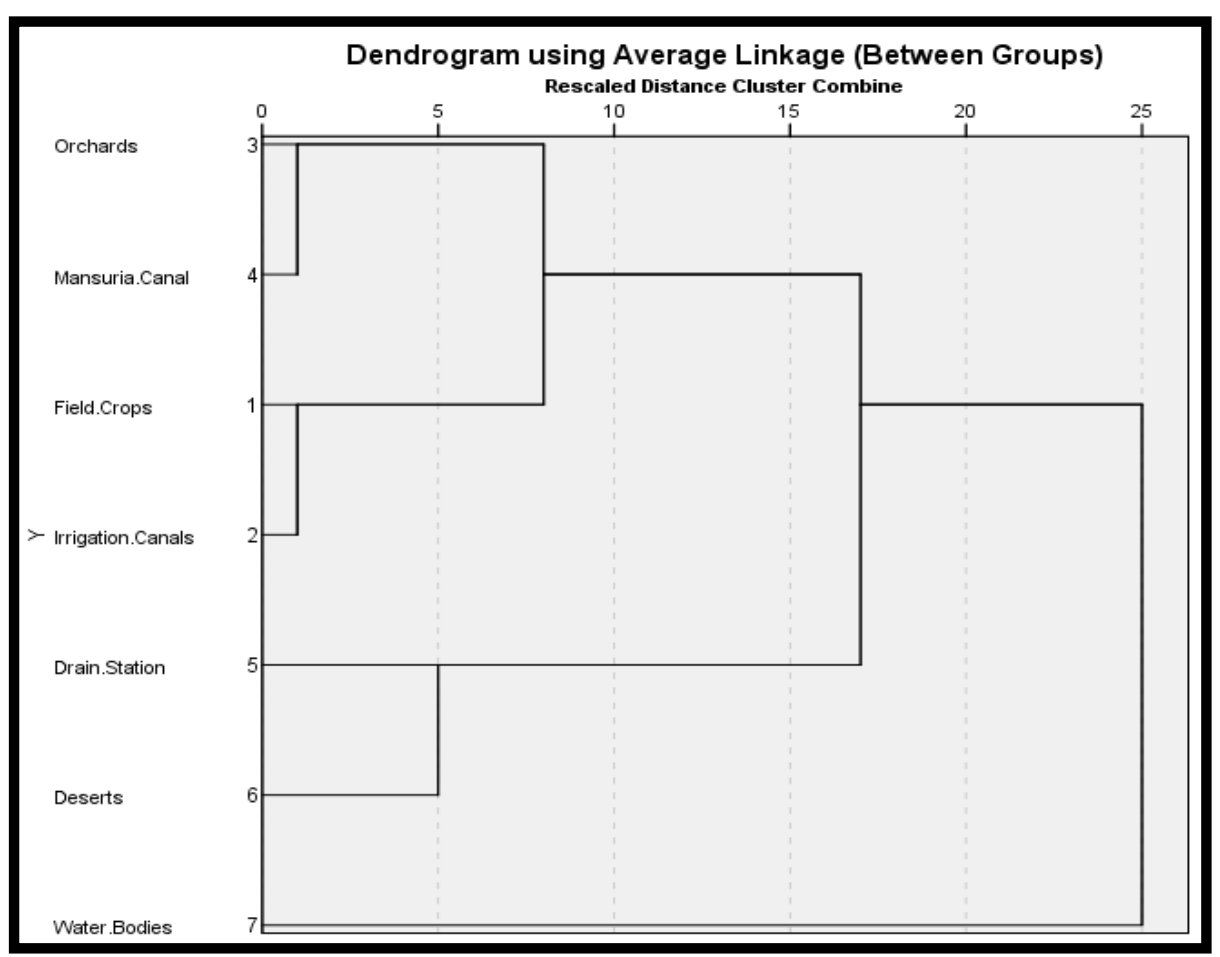

Fig. (4): Dendrogram showing the degree of similarity among the studied habitats in Abu Rawash area.

of Abu Rawash area. This represented 55\%, followed by chamaephytes (18\%), phanerophytes (7\%) and hemicryptophytes (6\%). Moreover, each of geophytes, helophytes and hydrophytes represented by $4 \%$; while nanophanerophytes were $2 \%$ (Fig. 6).

\section{4. Chorological ratios}

\section{4. 1. Mono-regional species ratios:}

The mono-regional species ratios of the studied species outlined in Fig. 8 revealed that each of Saharo-Sindian and Sudano-Zambezian regions represented $4 \%$ while Mediterranean region was $3 \%$. On the whole, the total ratios of mono-regional species were $11 \%$.

\section{4. 2. Bi-regional species ratios}

The bi-regional species dominated by (Mediterranean and Irano-Turanian) regions constituted 6\%, followed by Saharo-Sindian and Sudano-Zambezian (5\%) and Irano-Turanian and Saharo-Sindian 3\% while Mediterranean and Saharo-Sindian) regions were $1 \%$. Moreover, the total ratios of bi-regional species were $15 \%$ (Fig. 9).

\section{4. 3. Tri-regional species ratios}

In addition the tri-regional species dominated Mediterranean, Irano-Turanian and Euro-Siberian regions constituted 5\% followed by Mediterranean, Irano-Turanian and SaharoSindian regions (4\%) while each of Mediterranean, Euro - Siberian and Saharo-
Sindian and Irano-Turanian, Saharo-Sindian and Sudano-Zambezian regions was 3\% (Fig.7). On the whole, the total ratios of tri-regional species was $15 \%$ (Fig.10).

\section{4. 4. Pluri-regional species}

It was obvious that cosmopolitan have the highest ratio (23\%) followed by pantropical $(16 \%)$, palaeotropical $(13 \%)$ then cultivated species $(7 \%)$. On the whole, the total ratios of pluri-regional species were (59\%) (Fig.11).

\section{5. Habitats species ratios}

It was obvious that the annual species constituted the main bulk of the recorded species at the following habitats: field crops, irrigation canals, orchards, drain station and Mansuria Canal. The weed of field crops represented $88.24 \%$ followed by irrigation canals (80\%), drain station (65.79), orchards $(65.22 \%)$ and Mansuria Canal (56.25\%). On the other hand the perennial species constituted the main ratios at water bodies and deserts habitats, where they represented $100 \%$ and $51.28 \%$ respectively. The biennial species were represented by $4.35 \%$ at orchards habitats. Moreover, each of shrubs and trees were represented by $2.94 \%$ at field crops, $3.33 \%$ at irrigation canal and $6.25 \%$ at Mansuria Canal habitats, while they did not record at water bodies' habitats (Fig. 12). 


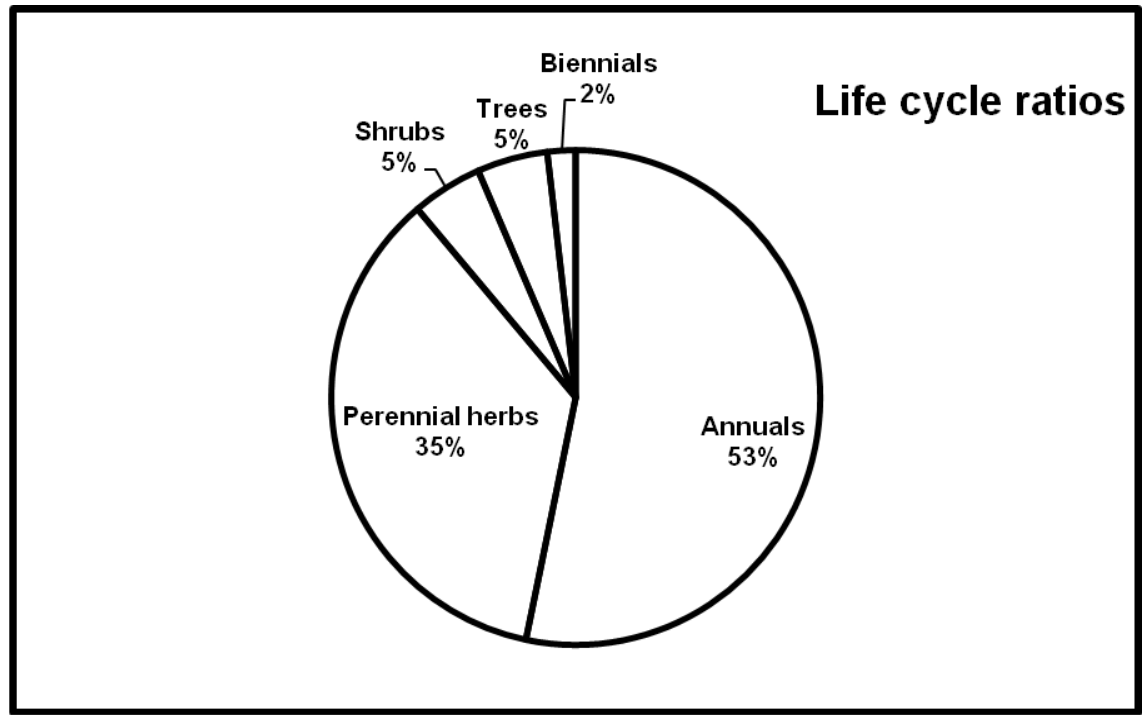

Fig. (5): Spectrum showing the life cycle ratios of the recorded species among the studied habitats in Abu Rawash area.

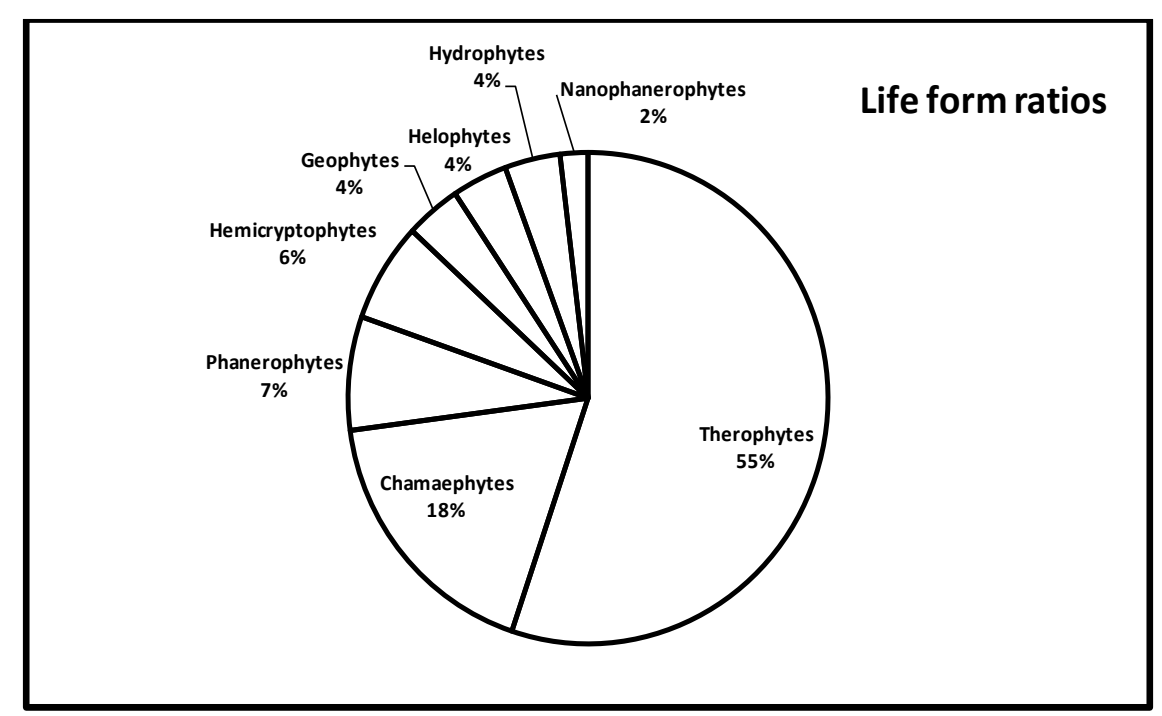

Fig. (6): Spectrum showing the life form ratios of the recorded species among the studied habitats in Abu Rawash area.

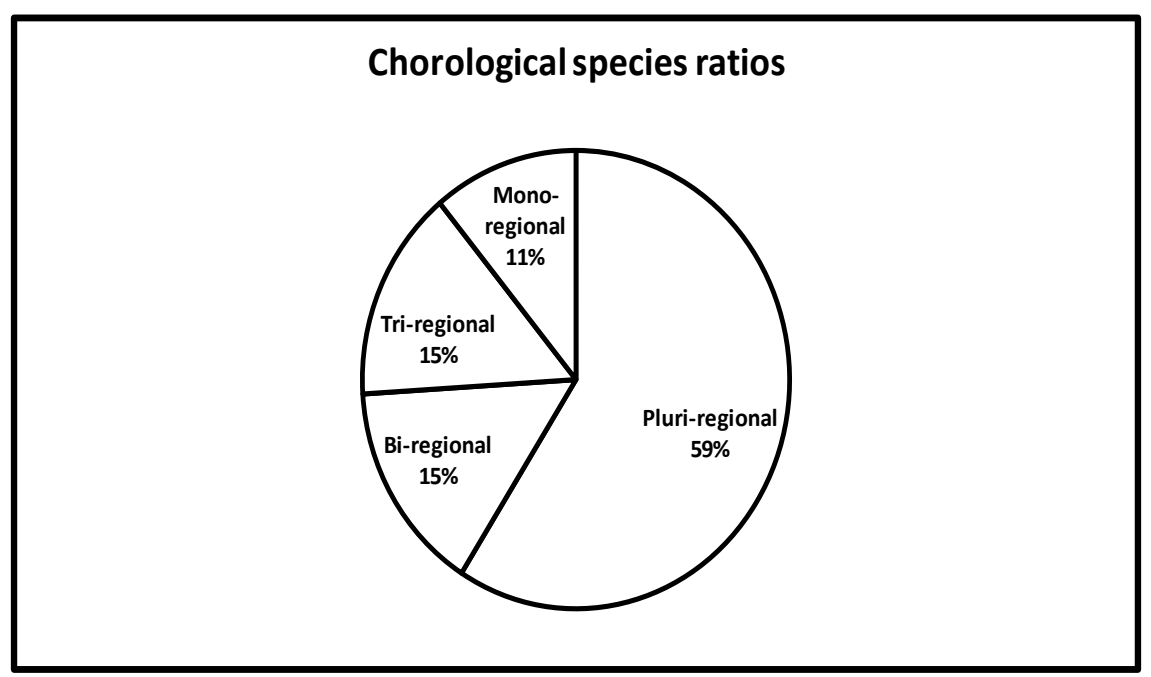

Fig. (7): Spectrum showing the chorological species ratios of the recorded species among the studied habitats in Abu Rawash. 


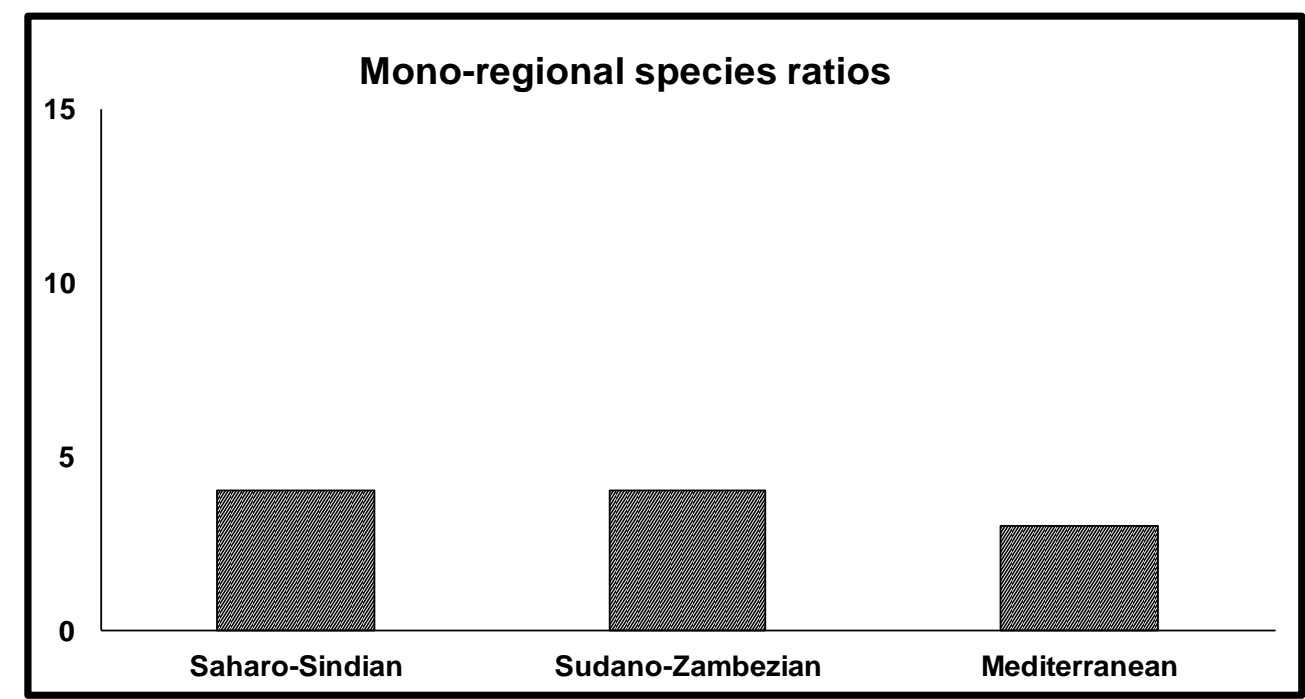

Fig. (8): Histogram showing the mono-regional species ratios of the recorded species among the studied habitats in Abu Rawash area.

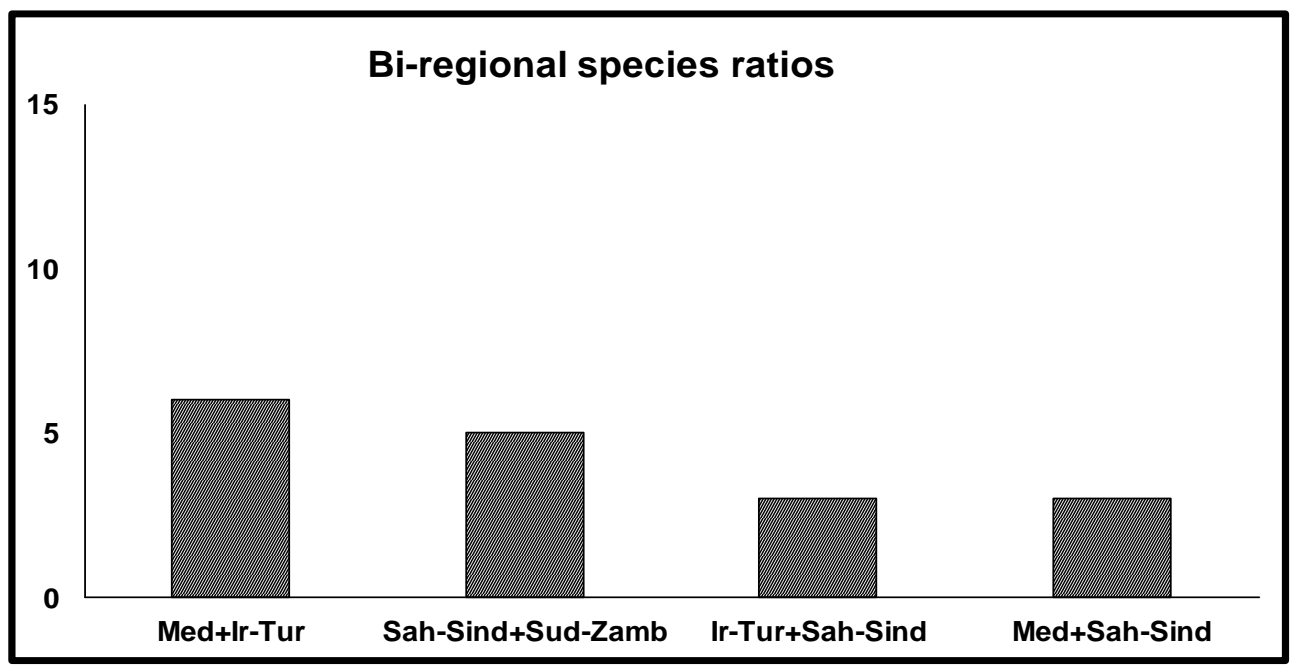

Fig. (9): Histogram showing the bi-regional species ratios of the recorded species among the studied habitats in Abu Rawash area. (Med= Mediterranean, Ir-Tur = Irano-Turanian, Sah-Sind = Saharo-Sindian, Sud-Zamb = Sudano-Zambezian).

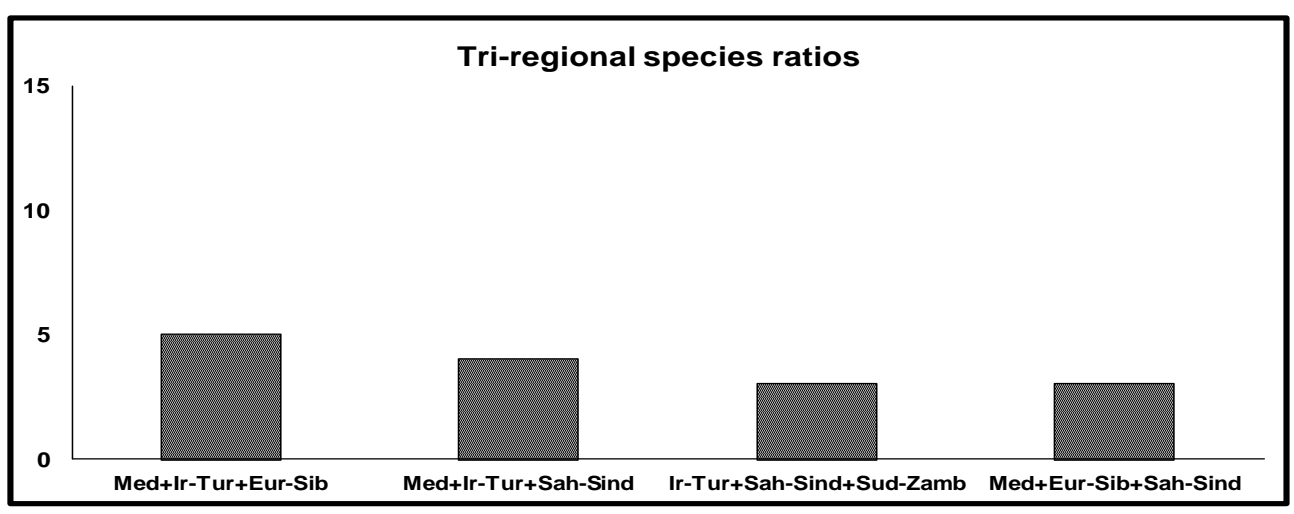

Fig. (10): Histogram showing the tri-regional species ratios of the recorded species among the studied habitats in Abu Rawash area. (Med= Mediterranean, Ir-Tur = Irano-Turanian, Eur-Sib = Euro-Siberian, Sah-Sind = SaharoSindian, Sud-Zamb = Sudano-Zambezian). 


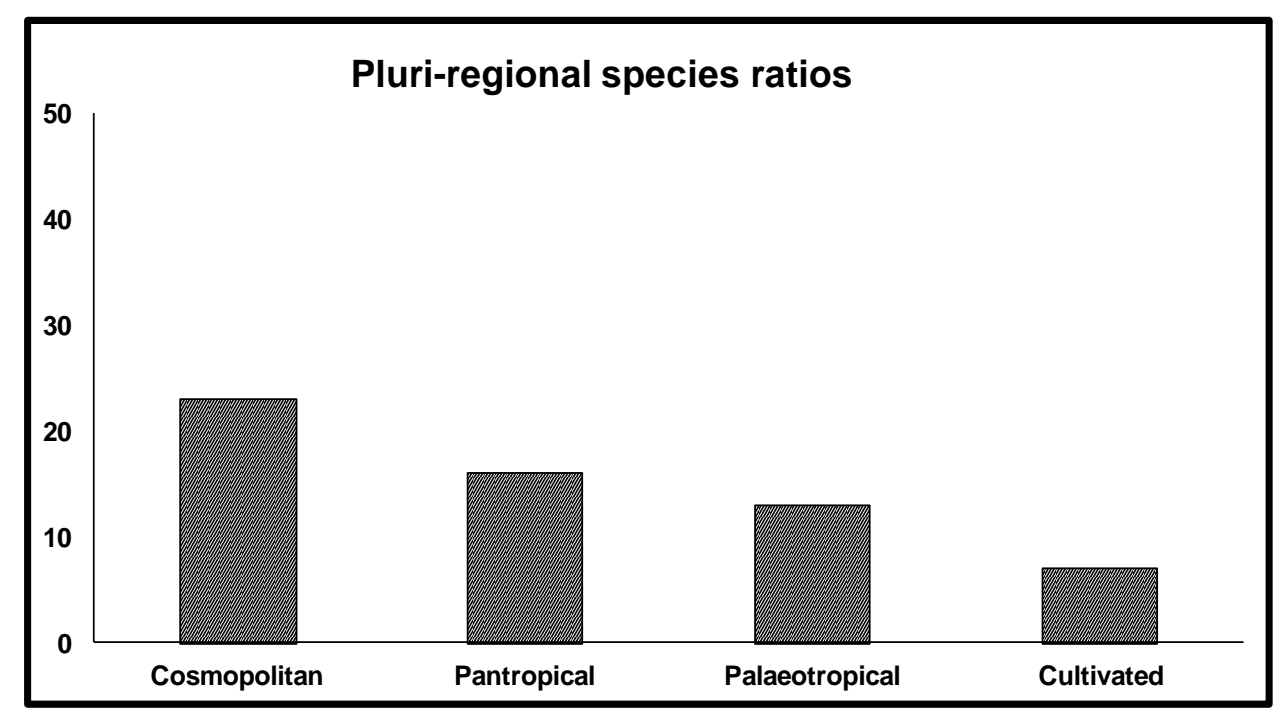

Fig. (11): Histogram showing the pluri-regional species ratios of the recorded species among the studied habitats in Abu Rawash area.

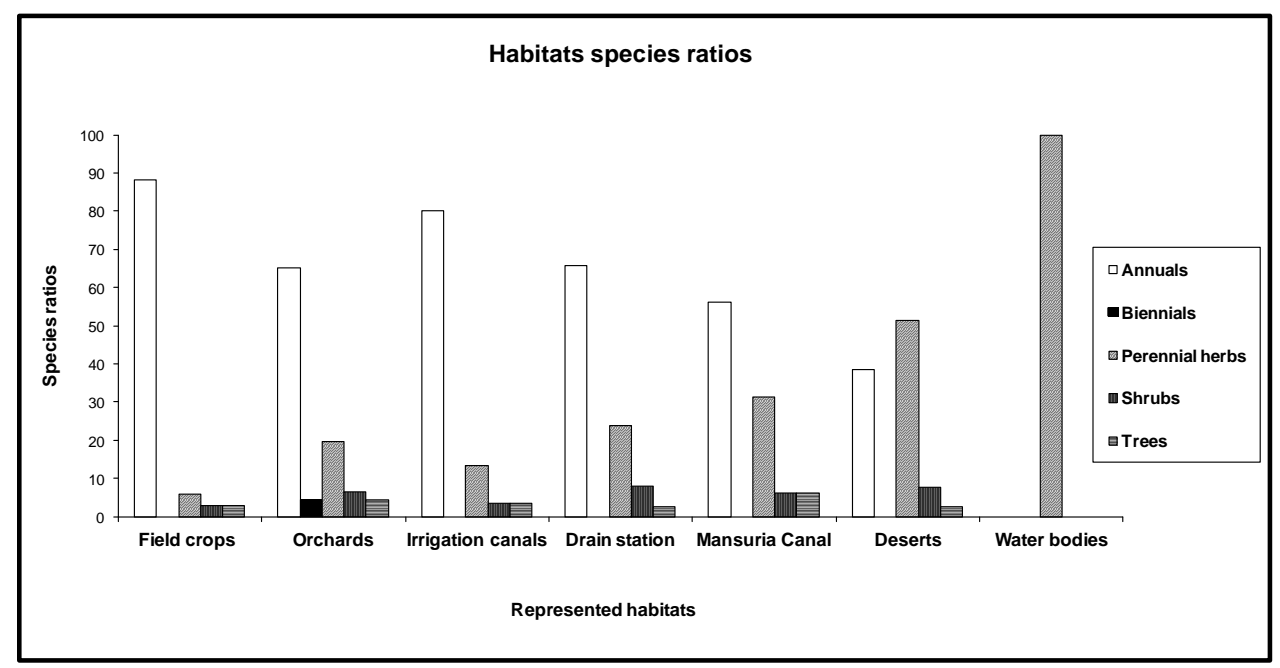

Fig. (12): Histogram showing the habitats species ratios of the recorded species among the studied habitats in Abu Rawash area.

Table (3): Proximity matrix showing the similarity values of species distributed among the studied habitats in Abu Rawash area.

\begin{tabular}{|c|c|c|c|c|c|c|c|}
\hline \multirow{2}{*}{ Hapitats } & \multicolumn{7}{|c|}{ Proximity Matrix } \\
\cline { 2 - 8 } & $\begin{array}{c}\text { Field } \\
\text { crops }\end{array}$ & $\begin{array}{c}\text { Irrigation } \\
\text { canals }\end{array}$ & Orchards & $\begin{array}{c}\text { Mansuria } \\
\text { Canal }\end{array}$ & $\begin{array}{c}\text { Drain } \\
\text { station }\end{array}$ & $\begin{array}{c}\text { Water } \\
\text { Deserts } \\
\text { bodies }\end{array}$ \\
\hline Field crops & 1.000 & & & & & & \\
Irrigation canals & 0.529 & 1.000 & & & & & \\
Orchards & 0.415 & 0.477 & 1.000 & & & & \\
Mansuria Canal & 0.304 & 0.362 & 0.543 & 1.000 & & & \\
Drain station & 0.197 & 0.203 & 0.264 & 0.369 & 1.000 & & \\
Deserts & 0.056 & 0.097 & 0.148 & 0.213 & 0.451 & 1.000 & \\
Water bodies & 0.000 & 0.000 & 0.000 & 0.017 & 0.000 & 0.000 & 1.000 \\
\hline
\end{tabular}


3.6.Major similarity among the studied habitats in Abu Rawash area

The data presented in Table (4) and dendrogram Fig.(4), showed that, the recorded species among seven represented habitats were separated into four groups (G1, G2, G3 and G4). Group one included field crops and irrigation canals habitats. The second group contained orchards and Mansuria Canal habitats. The third group included drain station and deserts habitats, while the forth group included water bodies' habitat. Moreover, orchards and Mansuria Canal habitats showed the highest degree of similarity value 0.543 followed by 0.529 between field crops and irrigation canal habitats, and 0.477 and (0.451) between (irrigation canals and orchards and drain station and deserts habitats; respectively.

\section{7. Major dissimilarity among the studied habitats in Abu Rawash area}

The data presented in Table (3) and dendrogram Fig. (4) showed that there is no degree of similarity between the species of water bodies habitats and the other habitats namely, field crops, irrigation canals, orchards, orchards, drain station and deserts habitats.

Table (4): Grouping of the studied habitats based on the numerical analysis of 107 species recorded in Abu Rawash area

\begin{tabular}{|c|c|}
\hline Studied habitats & Groups \\
\hline $\begin{array}{l}\text { Field crops and irrigation } \\
\text { canals }\end{array}$ & G 1 \\
\hline Orchards and Mansuria Canal & G 2 \\
\hline Drain station and deserts & G 3 \\
\hline Water bodies & G 4 \\
\hline
\end{tabular}

\section{DISCUSSION}

A total of 107 species belonging to 92 genera and 33 families of the vascular plants were recorded. The recorded plant species represent about $5.02 \%$ of the Egyptian flora (Boulos, 1995 and 2009). The three major distributed families were Poaceae, Compositae and Brassicaceae. They comprised 44 taxa (41\%) of the total recorded species. These families were reported earlier by Mashaly et al., (2009), Hamed et al. (2012), Azer (2013) and Amer et al. (2015) as the most frequent families in the studied areas. The families with the highest richness recorded by this study were compatible with the data of Quezel (1978) who reported that, Poaceae, Compositae, Brassicaceae, Chenopodiaceae and Leguminosae are among the most common families in the Mediterranean North African flora. Similar conclusion has been reached by Shaheen (2002) and Abd El-Ghani and Fawzy (2006).

Dominance of the perennial species and limited number of the annual ones at the desert habitats may be attributed to the severe environmental factors mainly aridity and salinity that characterize the study area. Heneidy and Bidak (2001) and Abd El-Ghani et al. (2011) reported the short life cycles of annual species probably lead to the frequent occurrence during the favorable seasons which supports the present conclusion. In accordance with this report, the composition of life cycle revealed that, perennial herbs represent majority of recorded species in the desert and water bodies' habitats, whereas annual species are the most common in field crops, orchards, irrigation canals, drain station and Mansuria Canal habitats. Abd El-Ghani and Abd El-Khalik (2006) explained these relationships based on the extensive root systems of the perennial species that are capable of utilizing water stored at different soil depths. These explanations are supported by the present investigation based on the studied species among different habitats. On the other hand, the low number of shrubs and trees, in the current study related to the high intensity of disturbance due to agricultural activities in the field crops and orchards, this fact also reported by Kim et al. (2002), Abd El-Ghani et al .(2013) and Amer et al. (2015).

The dominant life forms in the studied area were therophytes species followed by chamaephytes, phanerophytes then hemicryptophytes. As in the whole Egyptian flora, the therophytes were the most common life form (Hassib, 1951). Similar observation was cited by El-Ghareeb and Rezk (1989). Moreover, Heneidy and Bidak (2001) mentioned that the dominance of therophytes response to the hot dry climate, topographic variation and biotic influence.

The chorological ratios of the recorded taxa showed that cosmopolitan had the highest contribution followed by pantropical then palaeotropical. This current study confirmed by Amer et al. (2015). Moreover, the widely distributed species belong to cosmopolitan, pantropical and palaeotropical chorotypes constituted $52 \%$ in the studied area. This 
indicated that the floristic structure of the study area was affected by human disturbances (Shaltout and El-Fahar, 1991; Abd El-Ghani et al., 2011 and Amer et al., 2015). Also the current study was supported by (El-Hadidi, 1993) who concluded that the major percentage of the weed flora of Egypt is represented by cosmopolitan, pantropical and palaeotropical taxa. The presence of species related to different chorotypes categories was related to the position of Egypt at the border line between the Asiatic and African continents (Amer et al., 2015). Also, El-Hadidi, (1993) mentioned that the natural vegetation of Egypt belongs to Saharo-Sindian; Sudano-Zambezian; Mediterranean and IranoTuranian regions.

\section{Conclusions}

In this study, the analysis of floristic variations concluded that, the recorded species included 107 represents 92 genera and 33 families. The largest families were Poaceae followed by Compositae and Brassicaceae. Three species were recorded from each of Cyperaceae, Malvaceae and Zygophyllaceae and two species from each of Amaranthaceae, Apiaceae, Apocynaceae, Plantaginaceae and Salicaceae. Moreover, high degree of monotypism was observed. The dominant life cycle species were annuals followed by perennials species. The chorological ratios showed that cosmopolitan taxa had the highest contribution followed by pantropical and palaeotropical. The cluster analysis divided the studied habitats into four groups. In addition to, orchards and Mansuria Canal habitats showed the highest degree of similarity. On the other hand, there was no similarity between species of water bodies' habitat and species of the other habitats except with Mansuria Canal. On the whole, the anthropogenic factors are operating together and reducing the chances of formation of new vegetation structure. This unique area needs an urgent protection.

\section{REFERENCES}

Abd El-Ghani M.M. and Abd El-Khalik L. (2006). Floristic diversity and phytogeography of the Gebel Elba national park, south-east Egypt. Turk. J. Bot., 30: 121-136.

Abd El-Ghani M.M. and Fawzy A.M. (2006). Plant diversity around springs and wells in five oases of the Western Desert, Egypt. Int`' 1. J. of Agric. and Biol., 8: 249-255.
Abd El-Ghani M.M., Bornkamm R., El-Sawaf N. and Turkey H. (2011). Plant species distribution and spatial habitat heterogeneity in the landscape of urbanizing desert ecosystem of Egypt. Urban Ecosyst., 14: 585-616.

Abd El-Ghani M.M., Soliman A.T., Hamdy R. and Bennoba E. (2013). Weed flora in the reclaimed lands along the northern sector of the Nile Valley in Egypt. Turk. J. Bot., 37: 464-488.

Alaily F., Bornkamm R., Blume H.P., Kehl H. and Zielinski H. (1987). Ecological investigations in the Gilf Kebir (SWEgypt). Phytocoenol., 15: 1-120.

Amer W., Soliman A. and Hassan W. (2015). Floristic composition of Nile islands in Middle Egypt with special reference to the species migration route. J. Amer. Sci., 11 (6): 14-23.

Angiosperm Phylogeny Group III (2009). An update of the Angiosperm Phylogeny Group classification for the orders and families of flowering plants: APG III". Bot. J. Linnean Soc., 161 (2): 105-121.

Azer S.A. (2013). Study on the plant diversity in Saqqara region, Giza, Egypt. Egyptian J. Biotech., 45: 1-17.

Bornkamm R. (1986). Flora and vegetation of some small oases in South of Egypt. Phytocoenol., 14(2): 275-284.

Bornkamm R. and Kehl H. (1989). Landscape ecology of the Western Desert of Egypt. J. Arid Environ., 17: 271-277.

Bornkamm R. and Kehl H. (1990). The plant communities of the Western Desert in Egypt. Phytocoenologia, 19 (2): 149-231.

Boulos L. (1980). Journey to the Gilf Kebir and Uweinat, southwest Egypt, IV. Botanical results of the expedition. Geographical J., 146: 51-93.

Boulos L. (1982a). Flora of Gebel Uweinat and some neighboring regions of southwestern Egypt. Candollea, 37: 257-276.

Boulos L. (1982b). The flora of Gebel Uweinat and neighboring regions, southwest Egypt. In: El-Baz, F. and Maxwell, T. A. (eds.) NASA Landforms of Southwest Egypt: a basis for comparison with Mars ,ppCR3611,Washington, D. C. Desert.,341 -347.

Boulos L. (1995). Flora of Egypt Checklist. Al Hadara Publishing, Cairo, Egypt. 10p.

Boulos L. (1999). Flora of Egypt. Vol. 1 (Azollaceae - Oxalidaceae). Al-Hadara Publishing, Cairo, Egypt. 24pp. 
Boulos L. (2000). Flora of Egypt. Vol. 2 (Geraniaceae - Boraginaceae). Al-Hadara Publishing, Cairo, Egypt. 36p.

Boulos L. (2002). Flora of Egypt. Vol. 3 (Verbinaceae - Compositae). Al-Hadara Publishing, Cairo, Egypt. 34p.

Boulos L. (2005). Flora of Egypt. Vol. 4 (Monocotyledons: Alismataceae Orchidaceae). Al Hadara Publishing, Cairo, Egypt.102p.

Boulos L. (2008). Flora and vegetation of the deserts of Egypt. Flora Mediterranean, 18: 341-359

Boulos L. (2009). Flora of Egypt checklist (Revised annotated edition). Al Hadara Publishing, Cairo, Egypt. Pp.16.

Boulos L. and Barakat H.N. (1998). Some aspects of the plant life in the Western Desert of Egypt. J. Union Arab Biol., Cairo 5 (B): 79-94.

Chase M.W. and Reveal J.L. (2009). A phylogenetic classification of the land plants to accompany APG III". Bot. J. Linnean Soc., 161 (2): 122-127.

El-Ghareeb R. and Rezk R.M. (1989). A preliminary study on the vegetation of the coastal land at Bousseli (Egypt). Kuwait J. Sci., 16: 115-127.

El-Hadidi M.N. (1993). Natural vegetation. In the agriculture in Egypt, G.M. Craig (ed.), Oxford University Press., UK 39p.

Faris M.I. and Soliman S.M. (1961). Petrology of the dolomite rocks of Abu Rawash and its consideration to possible petroleum reservoirs in other parts of Egypt. Third Arab Petroleum Congress, Alexandria, Pp., Egypt.1-30.

Feinbrun-Dothan, N. (1978). Flora Palaestina, (Part three). The Israel Academy of Science and Humanities, Israel Jerusalem Academic Press, Jerusalem, Israel, 90p.

Feinbrun-Dothan, N. (1986). Flora Palaestina, (Part four). The Israel Academy of Science and Humanities, Israel, Jerusalem Academic Press, Jerusalem. Israel, 123p.

Hamed S.T., Sheded M.G. and Owis M. (2012). Floristic composition of some riverian islands at Qena governorate - Egypt. Egypt. J. Bot., $2^{\text {nd }}$ international conference, Minia University, 299- 322.

Hassib M. (1951). Distribution of plant communities in Egypt. Bull.of Fac. Sci., Univ. Fouad 1, Cairo, Egypt, 29: 59-261.

Haston E., Richardson J.E., Stevens P.F., Chase M.W. and Harris D.J. (2009). The Linear
Angiosperm Phylogeny Group (LAPG) III: a linear sequence of the families in APG III. Botanical Journal of the Linnean Society, 161, 128-131.

Heneidy S.Z. and Bidak L.M. (2001). Multipurpose plant species in Bisha, Asir region, Southwestern Saudi Arabia. J. King Saud Univ.,Sci., 13: 11-26.

Kehl H. (1987). Zonation and establishment of vegetation in selected uninhabited Egyptian and Sudanese oases. Catena Supplement, 14: 275-290.

Kehl H. and Bornkamm R. (1993). Landscape ecology and vegetation units of the Western Desert of Egypt. Catena Supplement, 26: 155-178.

Kerdany M. and Cherif O.H. (1990). MesozoicIn: The Geology of Egypt,Said R. (ed.): Balkema Publication, Brookfield, Rotterdam, Boston, USA, 407-438.

Kim Y.M., Zerbe S. and Kowarik I. (2002). Human impact on flora and habitats in Korean rural settlements. J. Preslia, 74:409-419.

Mansour A.S.M. (2004). Diagenesis of Upper Cretaceous Rudist Bivalves, Abu Rawash Area, Egypt: A Petrographic Study. Geol. Croatica, 27 (1): 55-66.

Mashaly I.A., El-Habashy I.E., El-Halawany E.F. and Omar G. (2009). Habitat and plant communities in the Nile delta of Egypt II. Irrigation and drainage canal bank habitat. Pak. J. Biol. Sci., 12: 885895.

Quézel P. (1978). Analysis of the flora of Mediterranean and Saharan Africa. Ann. Missouri Bot. Garden, 65: 479-534.

Raunkiaer C. (1934). The plant life forms and statistical plant geography. Clarendon Press, Oxford, UK, Pp. 10.

Salman A.B., Howari F.M., El-Sankary M.M., Wali A.M. and Saleh M.M. (2010). Environmental impact and natural hazards on Kharga Oasis monumental sites, Western Desert of Egypt. J. Afr. Earth Sci., 341-353.

Shaheen A.M. (2002). Weed diversity of newly farmed land on the southern border of Egypt (Eastern and Eastern shores of Lake Nasser). Pak. J.Biol. Sci., 5: 602-608.

Shaltout K.H. and El-Fahar R. (1991). Diversity and phenology of weed communities in the Nile Delta region. J.Veg. Sci., 2:385390. 
SPSS (2013). IBM SPSS Statistics for Windows, Version 22.0. Armonk, NY: IBM Corp., USA. 25p.

Täckholm V. and Drar M. (1950). Flora of Egypt., 2. Bull. . Fac. . Sci...Cairo University. 12p.

Täckholm V. and Drar M. (1954). Flora of Egypt. 3. Bull. .Fac.y of Sci., Cairo University. 22p.

Täckholm V. and Drar M. (1969). Flora of Egypt. 4. Bull. .Fac.of Sci.,.Cairo University. 10p.

Täckholm V. and Täckholm G. (1941). Flora of Egypt. 1. Bull. Fac. of Sci., Cairo University. 25p.

Täckholm V. (1974). Students' Flora of Egypt. $2^{\text {nd }}$ edition. Published by Cairo Univ. Herbarium Printed by Cooperative Printing Company Beirut,Lebanon,Pp.42.
Wickens G.E. (1976). The flora of Jabal Marra (Sudan Republic) and its Geographical Affinities. Kew Bull., Additional Series V. London: HMSO. Pp.13

Zahran M.A. and El-Amier Y.A. (2014). Ecology and establishment of fiber producing taxa naturally growing in the Egyp. deserts. Egyp. J. Basic and App. Sci., 1(3-4): 144-150.

Zohary M. (1966). Flora Palaestina (Part one). The Israel Academy of Science and Humanities, Israel Jerusalem Academic Press, Jerusalem, Israel,Pp. 15.

Zohary M. (1987). Flora Palaestina (Part two). The Israel Academy of Science and Humanities, Israel Jerusalem Academic Press, Jerusalem Israel,Pp. 55.

$$
\begin{aligned}
& \text { دراسة على التتوع النباتى فى منطقة أبورواش ، الجيزة، مصر } \\
& \text { صفوت أمين عازر } \\
& \text { قسم بحوث الفلورة وتصنيف النباتات ـ معطة بحوث البساتين - مركز البحوث الزراعية ـ الجبزة - مصر }
\end{aligned}
$$

تهذف هذه الدراسة الى القاء الضوء على تنوع النباتات البرية فى سبعة بيئات بمنطقة الدراسة و هي (المحاصيل

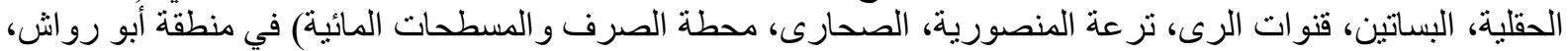

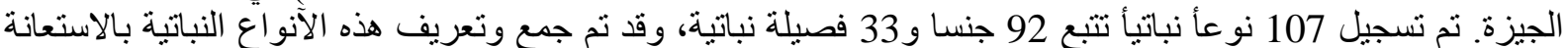

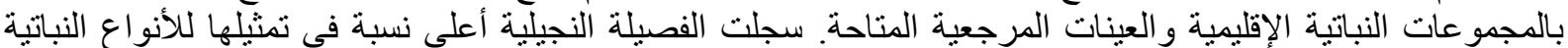

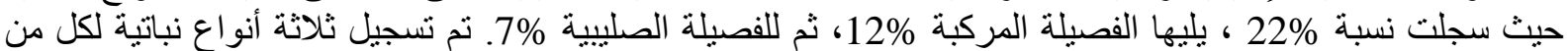
الفصيلة السعدية، الخبازية و الرطريطية ونوعين لكل من فصيلة عرف الديك، الفصيلة الخيمية، الفصيلة الدفلية، فصيلة

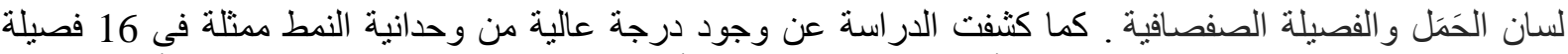

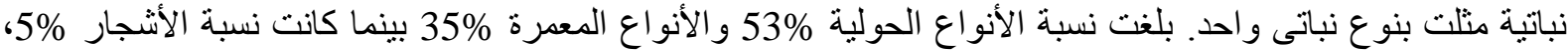

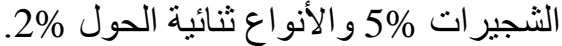

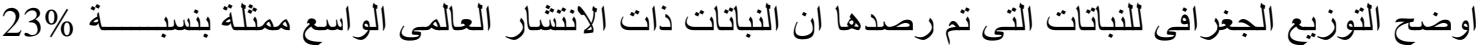

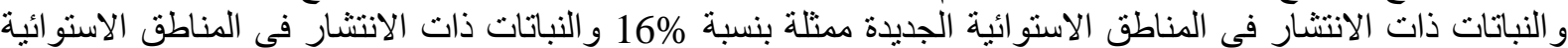

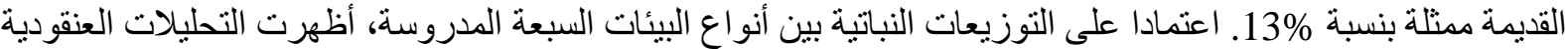
درجة كبيرة من التشابه بين نباتات البيئات المدروسة، حيث قسمت التبات الأنواع حسب هذه التبات التحليلات إلى التى أربعة مجمو عات:

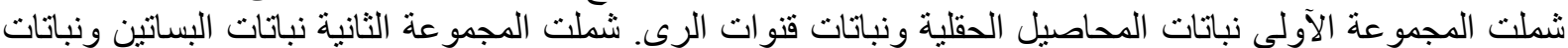

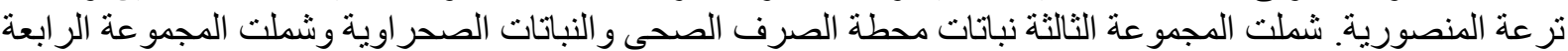

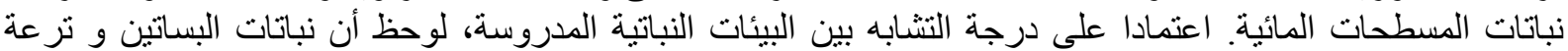

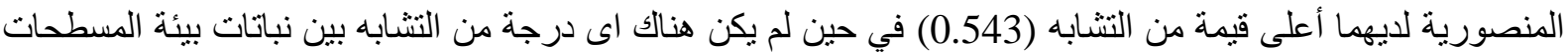

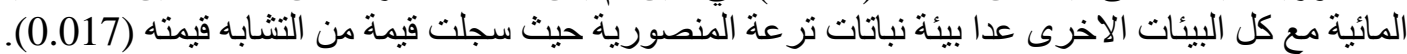
المجلة العلمية لكلية الزراعة - جامعة القاهرة ـ المجلا (68) العدد الأول (يناير 2017): 